International Journal of Engineering \& Technology, $7(2.23)(2018) 111-113$
International Journal of Engineering \& Technology
SPC
Website www.sciencepubco.com/index.php/IJET
Research paper

\title{
Modeling of Radio-wave Propagation in Forest by the Method of Parabolic Equation
}

\author{
M.S. Mikhailov ${ }^{1}$, E.S. Malevich ${ }^{1 *}$, V.A. Permyakov ${ }^{1}$ \\ ${ }^{1}$ Department of Radio Engineering Devices and Antenna Systems, National Research University "Moscow Power \\ Engineering Institute”, Krasnokazarmennaya 14, 111250 Moscow, Russia \\ *Corresponding author E-mail:malevichmpei@gmail.com
}

\begin{abstract}
The influence of forests on the propagation of radio waves is an actual topic of research stimulated by practical tasks related to the operation of various types of communication, radar, radio navigation, geodesy, and remote sensing of the earth's surface. The dependence of the electric field strength modulus of the source above the ground surface is studied in the presence of an obstacle in the form of a forest massif. The main attention is paid to the analysis of the field within the forest with various parameters and forms of forest canopy. A new approach to the task is proposed for the forms of forest canopy, taking into account the type of forest: deciduous, coniferous or mixed. The effective dielectric permittivity is used as a parameter for specifying a forest massif. The propagation of radio waves is calculated using the parabolic equation method for a two-dimensional space model. The implemented methodology allows calculating sanitary protection zones and zones of building restriction, planning to plant or cut down forests for planning the propagation of radio waves taking this into account.
\end{abstract}

Keywords: Radio-wave propagation, Parabolic equation, Forest canopy, Lateral wave.

\section{Introduction}

When designing radio engineering systems, there is a need to estimate the magnitude of the electromagnetic field strength, taking into account the complex terrain topography, including uneven terrain, forest tracts, buildings, etc. The paper considers the propagation of radio waves using the parabolic equation method for a two-dimensional space model. The main factors influencing the propagation of radio waves are the surface of the earth, including large-scale irregularities, and the forest. The influence of refraction of radio waves in the troposphere is also possible. Because of the complexity of constructing the theoretical models of propagation of radio waves in the forest, a significant amount of work was done on the experimental determination of the attenuation of radio waves in the forest. Different variants of the location of the transmitting and receiving antennas are considered. The most detailed parameters were the location of both antennas inside the forest and the location of the transmitting antenna over the forest and the receiving antenna in the forest. Similar studies were conducted in different countries. The main attention is paid to the propagation of radio waves outside cities, because transmitting antennas of high power are accepted to be located in the territory taken out of the cities. The approach implemented in that work can be applied to the calculation of sanitary protection zones and construction restriction zones near the radio engineering systems.

\section{An overview of the parabolic equation}

The method of the parabolic equation (PE) for computing the propagation of radio waves in the troposphere was used even in
Leontovich's work in 1944 [1]. A detailed numerical realization for the two-dimensional case is presented in [2].

A parabolic equation for a two-dimensional space model can be obtained from the Helmholtz wave equation by simple transformations. The scalar Helmholtz equation for the vertical component of the electric field strength

$$
\Delta U+k_{0}^{2} \varepsilon U=0
$$

reduces to the equation for the function by substitution [2]:

$$
U(x, z)=W(x, z) \exp \left(-i k_{0} z\right)
$$

Assuming that the function is a slowly varying function of the coordinate as compared to a change in transverse coordinates, the resulting equation reduces to a parabolic equation:

$$
\frac{\partial^{2} W}{\partial x^{2}}-2 i k_{0} \frac{\partial W}{\partial z}+k_{0}^{2}[\varepsilon(x, z)-1] W=0
$$

A variant of the solution of the PE is considered. In the twodimensional case, the boundary condition is imposed on the upper and lower boundaries of the layered model for PE (3), on the surface of the earth at sliding angles of incidence of the wave this condition is simplified to the condition. The solution of a PE in a two-dimensional region of space is carried out using Fourier transforms in the section, where $z=$ const [3].

\section{Results of numerical simulation}

The geometry of the obstacle in the form of a plane-layered model is shown in Fig. 1. The obstacle is a four-layer forestry model with a given effective dielectric constant for each of the layers. Separate the layer of the canopy, the forms of which are used in the calculation, are shown in Fig. 2, 3. 


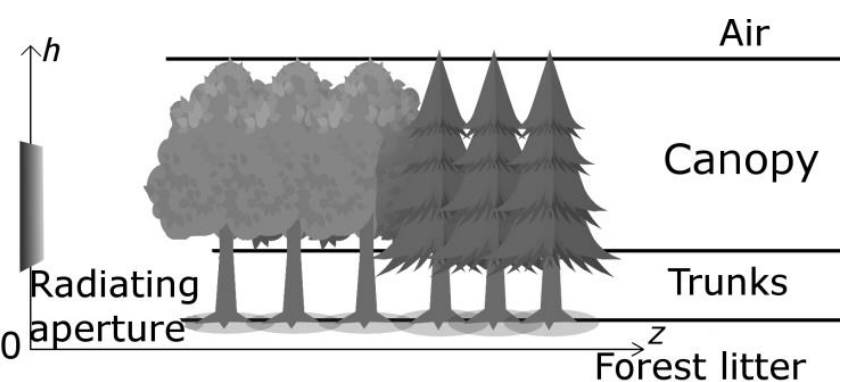

Fig. 1: Geometry of irregularities in the form of a forest massif on the Earth's surface.

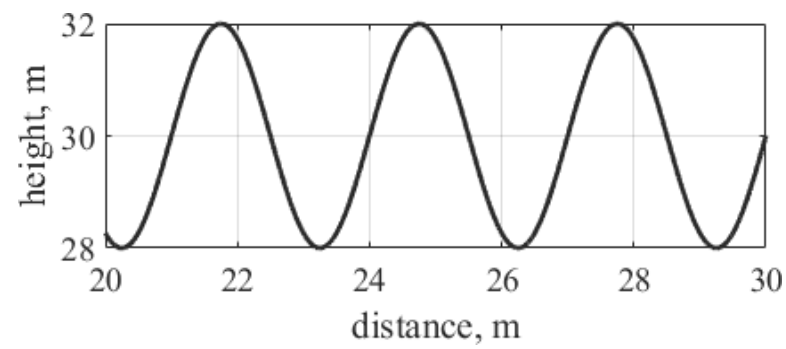

Fig. 2: Geometry of a sinusoidal canopy for a deciduous forest.

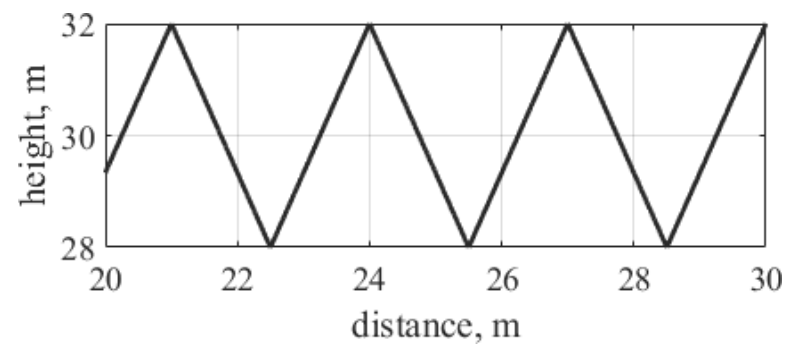

Fig. 3: Geometry of the wedge-shaped canopy for coniferous forest.

The source of the signal in the two-dimensional case is a single antenna with a predetermined radiation pattern. The beamwidth of the antenna pattern is $5^{\circ}$. The height of the antenna location above the ground is $8 \mathrm{~m}$. The radiation polarization is vertical. Operating frequency $600 \mathrm{MHz}$. The radiation regime is continuous.

Fig. 4-7 show the numerical results of calculating the field by the PE method in two-dimensional space. The initial distribution of the field was calculated in the approximation of geometric optics at a distance of $20 \mathrm{~m}$ from the antenna, taking into account the reflection from the Earth. Compared with the simulation results given in the report [4], where the lower limit of the PE was set as an ideally reflecting plane, a $20 \mathrm{~m}$ thick underlying surface with a given effective permittivity is used.

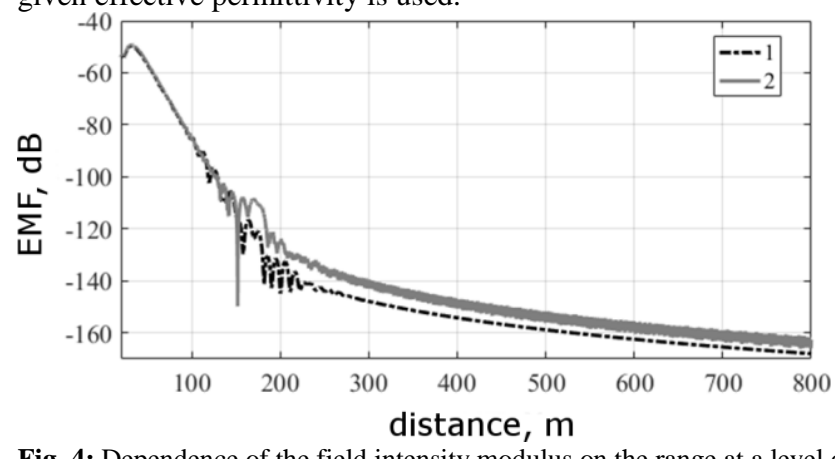

Fig. 4: Dependence of the field intensity modulus on the range at a level of $2 \mathrm{~m}$ above the ground for a deciduous forest of medium height of $20 \mathrm{~m}$. Curves 1-2 correspond to: 1) a flat canopy, 2) a sinusoidal canopy.

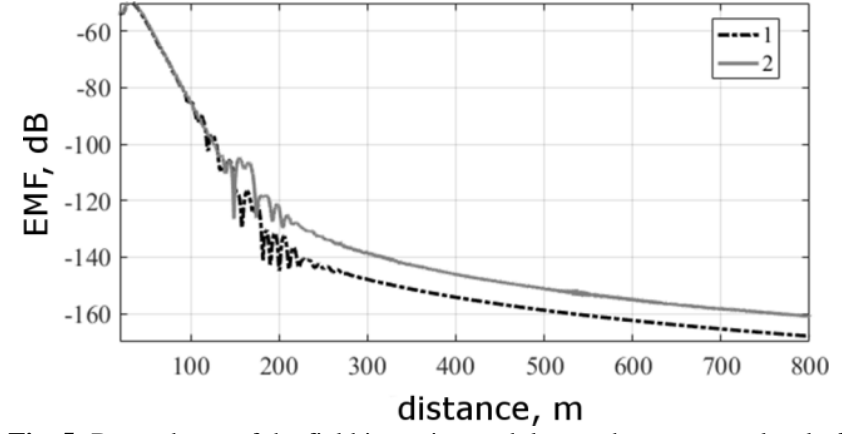

Fig. 5: Dependence of the field intensity modulus on the range at a level of $2 \mathrm{~m}$ above the ground for a coniferous forest of medium height of $20 \mathrm{~m}$. Curves 1-2 correspond to: 1) a flat canopy, 2) wedge shaped canopy.

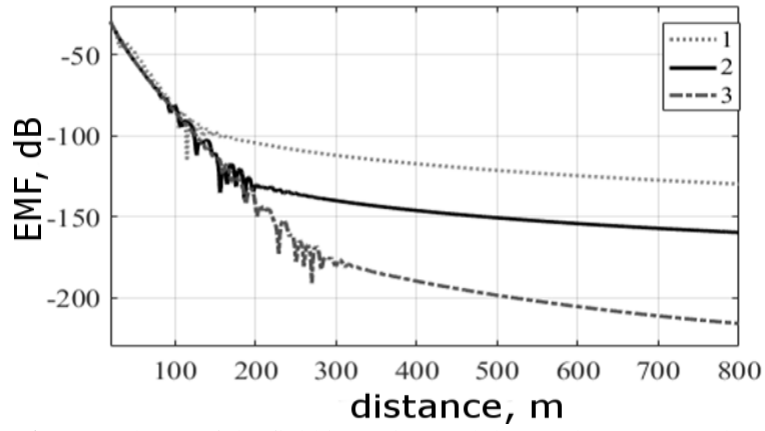

Fig. 6: Dependence of the field intensity modulus on the range at a level of $8 \mathrm{~m}$ above the ground for deciduous forest. Curves 1-3 correspond to: 1)

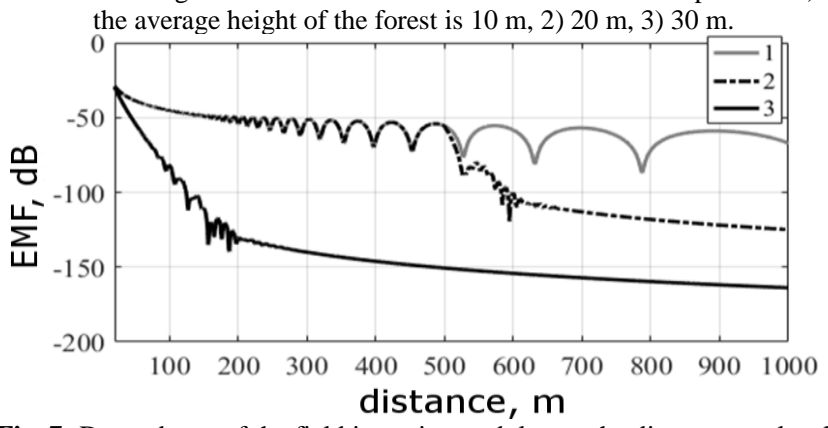

Fig. 7: Dependence of the field intensity modulus on the distance at a level of $8 \mathrm{~m}$ above the ground for three cases: 1) there is no forest, 2) deciduous forest begins $500 \mathrm{~m}$ from the antenna, 3) forest starts at $20 \mathrm{~m}$ from the antenna.

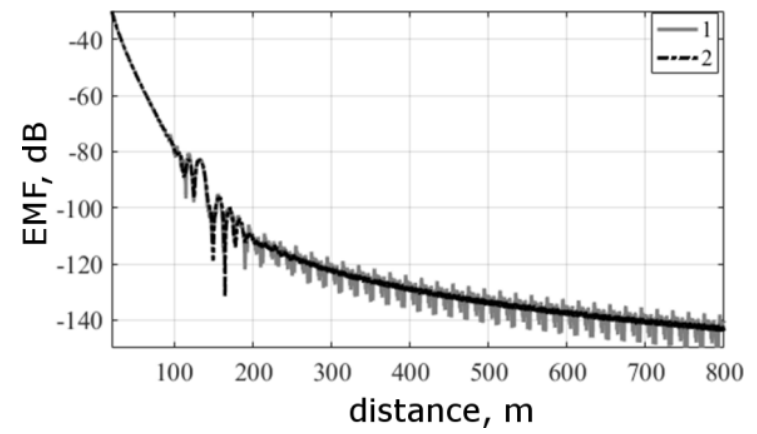

Fig. 8: Dependence of the field intensity modulus on the range at a level of $8 \mathrm{~m}$ above the ground for a coniferous forest of medium height of $20 \mathrm{~m}$. Curves 1-2 correspond to: 1) rarefied forest, 2) dense forest.

Calculations were carried out for flat and modulated canopies, sinusoidal and wedge-shaped at different heights at a level of 2 and $8 \mathrm{~m}$ from the ground at a frequency of $600 \mathrm{MHz}$. The effective dielectric permittivities of the canopy and trunks 1.1-i0.009 are given, for the underlying surface 4.1-i0.02 from [5]. The average distance between the trees is $3 \mathrm{~m}$. The change in the height of the edge of the canopy is set as $15 \%$ of the average height of the forest. All other parameters of the forest are shown in the figures.

Figure 4-5 clearly shows that to a range of $\sim 250 \mathrm{~m}$ the field distribution is oscillatory. This attenuation area corresponds to the exponential decay in the forward link between the antenna and the 
receiving point in the forest, the interference of the field is related to the reflection of the field from the underlying surface. At long ranges, communication is carried out in the mode of the lateral wave - signal attenuation follows the power law.

\section{Conclusion}

The realized plane-layer model of the forest, calculated using the two-dimensional method of the parabolic equation, makes it possible to evaluate the influence of the shape and characteristics of the forest canopy on the structure of the electromagnetic field. In the course of modeling, areas of influence of the lateral wave were identified, numerical characteristics of the dependence of the signal level on the height of the forest were determined. The method makes it possible to calculate the sanitary protection zones and the construction restriction zones near the powerful radio engineering systems taking into account the terrain profile in the presence of forest massifs.

\section{Acknowledgement}

This work was supported by the Ministry of Education and Science of the Russian Federation (project no. 8.9598.2017/BCh).

\section{References}

[1] Leontovich M.A., Izvestya Akademii Nauk SSSR, Seria Fizika, no. 8, p.16, 1944.

[2] Levy M., Parabolic Equation Methods for Electromagnetic Wave Propagation, ISBN: 0-85296-764-0, p. 336, 2000

[3] Permyakov V.A, Mikhailov M.S, Malevich E.S, Progress In Electromagnetics Research Symposium Abstracts, St. Petersburg, Russia, 22-25 May 2017, pp. 3754-3757.

[4] Mikhailov M.S, Permyakov V.A, Malevich E.S., Russian Physics Journal, no. 59, pp. 144-148, 2016.

[5] Popov V.I., Propagation of radio waves in forests, ISBN: 978-59912-0519-1, p. 392, 2015. 